\title{
Accurate and Real-Time Temperature Monitoring during MR Imaging Guided PTT
}

Xianfu Meng $t^{a}$, Boyu Zhang $\neq^{b}$, Yan Yi ${ }^{a}$, Hui Cheng ${ }^{a}$, Baoming Wang ${ }^{d}$, Yanyan

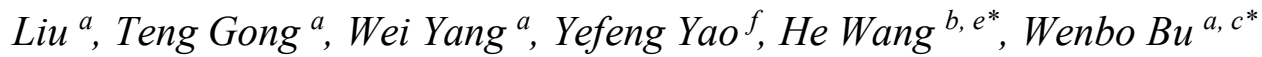

${ }^{a}$ Shanghai Key Laboratory of Green Chemistry and Chemical Processes, School of

Chemistry and Molecular Engineering, East China Normal University, Shanghai 200062, China

bInstitute of Science and Technology for Brain-Inspired Intelligence, Fudan University, Shanghai, China

${ }^{\mathrm{c} S t a t e}$ Key Laboratory of High Performance Ceramics and Superfine Microstructures, Shanghai Institute of Ceramics, Chinese Academy of Sciences, Shanghai, 200050, China

${ }^{\mathrm{d} S c h o o l}$ of Life Sciences, University of Technology Sydney, NSW, 2007, Australia

${ }^{\text {e} H u m a n ~ P h e n o m e ~ I n s t i t u t e, ~ F u d a n ~ U n i v e r s i t y, ~ S h a n g h a i, ~ C h i n a ~}$

fDepartment of Physics and Shanghai Key Laboratory of Magnetic Resonance, East China Normal University, Shanghai 200062, China

KEYWORDS: Photothermal therapy (PTT), T1-MRI, magnetic resonance temperature imaging (MRTI), temperature monitoring

ABSTRACT. 
Photothermal therapy (PTT) has been proved to be an efficient approach for cancer treatment. However, accurately monitoring the spatial distribution of photothermal transducing agents (PTAs) and mapping the real-time temperature change in tumor and peritumoral normal tissue remain a huge challenge. Here, we propose an innovative strategy to integrate $\mathrm{T} 1 \mathrm{weighted}$ imaging (T1WI) for precisely tracking PTAs with magnetic resonance temperature imaging (MRTI) for real-time monitoring temperature change in vivo during PTT. NaBiF 4 : Gd@PDA@PEG nanomaterials were synthesized with favorable T1-weighted performance to target tumor and localize PTAs. The extremely weak susceptibility $\left(1.04 * 10^{-6} \mathrm{emu} \mathrm{g}^{-1} \mathrm{Oe}^{-1}\right)$ of $\mathrm{NaBiF}_{4}$ : Gd@PDA@PEG interferes with the local phase marginally, which maintains the capability of MRTI to dynamically record real-time temperature change in tumor and peritumoral normal tissue. The time resolution is 19 seconds per frame, and the detection precision of temperature change is approximately as low as $0.1 \mathrm{~K}$. The approach combining anatomic and functional MRI to achieve accurate temperature monitoring during multi-mode MRI guided PTT holds significant potential for the clinical application.

Photothermal therapy (PTT) that employs photothermal transducing agents (PTAs) to convert absorbed light energy into heat for eradicating the tumor, is an effective method ${ }^{1-9}$. Numerous approaches based on nanoPTAs have been developed to treat tumor during the past decades. Because nanoPTAs can effectively target tumor, and 
the laser beam is further utilized to directly focus on tumor to improve the PTT accuracy ${ }^{10-19}$. However, the precise location of nanoPTAs in tumor and the accurate monitoring of real-time temperature in tumor and adjacent healthy tissue, are the main obstacles to acquire accurate PTT. Inevitably, when PTT is conducted, one side effect is the damage to peritumoral normal tissue caused by rapid heating, including disrupting cell components, changing membrane permeability, and even denaturing tissue structural proteins $\mathrm{s}^{20-22}$. Consequently, it is essential for PTT optimization to evaluate the delivery efficiency and spatial distribution of nanoPTAs, as well as the remote mapping and accurate monitoring of local temperature (i.e., maximizing thermal ablation of the tumor and minimizing damage to the healthy tissue).

Magnetic resonance imaging (MRI) has been widely employed in clinical noninvasive diagnosis and laboratory scientific research with high resolution and unlimited penetration depth in soft tissues ${ }^{23-27}$. Besides the commonly utilized anatomic techniques of T1/T2 weighted MRI, many functional MRI modalities have been developed and applied. Particularly, when MRI with multi-modal contrast agents (CAs) is conducted, anatomic MRI is capable of assessing the delivery and spatial distribution of CAs, contributing to more detailed anatomic information. Furthermore, functional MRI is qualified for sensitively monitoring the microenvironment of tumor. Recently, our group developed several multi-modal MRI CAs, through the marriage between anatomic MRI and functional MRI, which achieved accurate localization of tumor and detection of the tumor microenvironment $(\mathrm{pH}$, blood vessel $)^{28-30}$. Otherwise, few studies have been reported on combining anatomic MRI 
with functional MRI for real-time tumor temperature during PTT. Magnetic resonance temperature imaging (MRTI), through a proton resonance frequency (PRF) method, can real-time and accurately monitor the temperature change in the target area, which is based on the linear relationship between temperature and phase $\mathrm{s}^{31-35}$. However, the strong susceptibility brought by T2-MRI CAs will disturb the local phase, and further inhibit the accuracy of monitoring temperature ${ }^{36-37}$. Therefore, it is a tough challenge that reasonable designing MRI CAs confirms the distribution and acquires accurate real-time temperature mapping during PTT on a single MRI machine.

In this study, we proposed an effective strategy that integrated MRI (T1/MRTI) was applied to achieve the precise temperature monitoring during PTT on a single MR machine. Anatomic MRI (T1-MRI) was utilized to track nanoPTAs and capture detailed structural information in tumor. Meanwhile, functional MRI (MRTI) was conducted to accurately record the real-time temperature change in tumor and peritumoral normal tissue. As shown in Scheme 1, NaBiF4: Gd@PDA@PEG nanomaterials were constructed with outstanding photothermal conversion performance and T1-weighted capability. The distribution of nanoPTAs in tumor was recorded through T1-weighted MRI. More importantly, NaBiF4: Gd@PDA@PEG nanoparticles could further guarantee the accuracy of temperature mapping with intensely weak local phase susceptibility $\left(1.04 * 10^{-6} \mathrm{emu} \mathrm{g}^{-1} \mathrm{Oe}^{-1}\right)$. Therefore, MRTI was performed on an $11.7 \mathrm{~T}$ ultra-high field magnetic resonance machine to record the real-time and accurate temperature change in tumor and peritumoral normal tissues during PTT. The time resolution was 19 seconds per frame, and the detection 
accuracy of temperature change was approximately as low as $0.1 \mathrm{~K}$. These results clarified that integrated MRI could be competent for guiding PTT, which was of great significance to further promote clinical PTT.

\section{Synthesis and characterization of $\mathrm{NaBiF}_{4}:$ Gd@PDA@PEG}

$\mathrm{NaBiF}_{4}$ : Gd nanoparticles were synthesized by a simple method (Figure S1 and

Figure S2) ${ }^{38}$. Polydopamine (PDA) was then coated onto $\mathrm{NaBiF}_{4}$ : Gd nanoparticles to transform them into hydrophilic. PEG-NH 2 molecules were further grafted onto PDA through a Schiff base reaction, which further improved the biocompatibility ${ }^{39}$. Evidently, transmission electron microscopy (TEM) demonstrated that $\mathrm{NaBiF}_{4}$ : Gd@PDA@PEG nanoparticles were uniform and with a diameter around $120 \mathrm{~nm}$ (Figure 1A). And element mapping images confirmed the successful synthesis (Figure 1B-C). Furthermore, X-ray photoelectron spectroscopy (XPS) also showed that $\mathrm{NaBiF}_{4}: \mathrm{Gd} @ \mathrm{PDA} @ \mathrm{PEG}$ nanoparticles were successfully prepared (Figure 1D-I). Dynamic light scattering (DLS) indicated the hydration size of $\mathrm{NaBiF}_{4}$ : Gd@PDA@PEG was around 148.8 nm in water (Figure S3). Meanwhile, the results of X-ray diffraction (XRD) pattern also verified the successful synthesis of $\mathrm{NaBiF}_{4}$ : Gd and $\mathrm{NaBiF}_{4}$ : Gd@PDA@PEG nanoparticles. $\mathrm{Gd}^{3+}$ ions doping slightly changed the crystal form of $\mathrm{NaBiF}_{4}$. After coated with PDA and PEG, the resulting nanoparticles showed a little amorphous (Figure S4). Fourier transform infrared (FT-IR) spectra were performed to confirm the successful synthesis and modification of $\mathrm{NaBiF}_{4}: \mathrm{Gd}, \mathrm{NaBiF}_{4}: \mathrm{Gd} @$ PDA and $\mathrm{NaBiF}_{4}: \mathrm{Gd} @$ PDA@PEG nanoparticles 
(Figure S5). In addition, X-ray photoelectron spectroscopy (XPS) was conducted to characterize the compositions, which further exhibited the successful preparation of $\mathrm{NaBiF}_{4}: \mathrm{Gd}$ and $\mathrm{NaBiF}_{4}: \mathrm{Gd} @ \mathrm{PDA} @ \mathrm{PEG}$ nanoparticles (Figure S6).

To evaluate the photothermal performance, PDA coating content was firstly investigated. Thermogravimetric analysis (TG) and differential scanning calorimeter (DSC) showed PDA coating content was about 26\% accompanied by an exothermic reaction (Figure S7). From the results of UV-Visible-NIR absorption spectra (Figure S8A), NaBiF4: Gd@PDA@PEG had unique absorption feature in visible and near infrared (I) regions. Moreover, according to the equation of Lambert-Beer law $(\mathrm{A}=\varepsilon \mathrm{Lc})$, the extinction coefficient $(\varepsilon)$ was calculated to be $5.08 \mathrm{~L} \mathrm{~g}^{-1} \mathrm{~cm}^{-1}$ at the 808-nm wavelength (Figure S8B). As we all knew, polydopamine (PDA) had excellent photothermal converting performance, which could be adopted to kill tumor. $^{40-43}$ As shown in Figure S9A-B, NaBiF4: Gd@PDA@PEG also exhibited favorable photothermal performance. Therefore, the photothermal treatment efficiency of $\mathrm{NaBiF}_{4}$ : Gd@PDA@PEG was further studied on cells. From Figure S10A-C, the results firstly revealed that the nanoparticles could be well swallowed by cells. Even Bi concentration was as high as $800 \mathrm{ppm}$, the cell viability was still above 85\%, which meant $\mathrm{NaBiF}_{4}:$ Gd@PDA@PEG had low cytotoxicity and excellent biocompatibility (Figure S11A). After irradiation, a number of cells died and the viability was dependent on concentration (Figure S11B). In order to further investigate the photothermal ability for killing tumor cells, calcein-AM and propidium iodide (PI) were utilized to distinguish live and dead cells. The final results indicated 
most cells were overheated to death (Figure S12A-C), which also demonstrated the outstanding photothermal ability for tumor therapy.

\section{MRI performance and susceptibility in aqueous solution}

Doping $\mathrm{Gd}^{3+}$ ions brought favorable $\mathrm{T} 1$ weight-MRI performance. As shown in Figure 2A-B, T1 weighted-MRI performance of $\mathrm{NaBiF}_{4}$ : Gd@PDA@PEG nanoparticles showed a concentration dependent tendency. It was obvious that longitudinal relaxation time decreased gradually with the increase of $\mathrm{Gd}$ in T1-mapping images. The longitudinal relaxation rate was ultimately calculated as 1.2 $\mathrm{mM}^{-1} \mathrm{~s}^{-1}$, which indicated $\mathrm{NaBiF}_{4}$ : Gd@PDA@PEG had excellent T1 weight-MRI performance. MRTI, based on the proton resonance frequency (PRF) method, can real-time and accurately monitor temperature change, depending on the relationship between temperature and phase. However, the local phase was vulnerable to strong susceptibility. Accordingly, the magnetism of $\mathrm{NaBiF}_{4}$ : Gd@PDA@PEG was continued to be investigated. From the hysteresis loop, the magnetic susceptibility was calculated to be $1.04 * 10^{-6}$ emu $\mathrm{g}^{-1} \mathrm{Oe}^{-1}$, which demonstrated $\mathrm{NaBiF}_{4}$ : Gd@PDA@PEG had little magnetism and little effect on local phase (Figure 2C). Furthermore, the phase change along with temperature was further evaluated in agar. Through linear fitting between MR phase and temperature, the slope was about -0.1 radian/K (Figure 2D). The relationship between phase and temperature unchanged by various Gd concentrations, which illustrated the nanomaterials were no interference with local phase and temperature accuracy. In contrast, we also tested the phase 
change along with temperature in agar with $\mathrm{Fe}_{3} \mathrm{O}_{4}(\mathrm{Fe}, 30 \mathrm{ppm})$ (Figure S13). As expected, iron had a great influence on local phase, causing sharp phase fluctuations during the test, which demonstrated that iron-based nanomaterials (including $\mathrm{Fe}_{3} \mathrm{O}_{4}$ ) were not suitable for sensitive and accurate temperature measurement through phase change. In conclusion, $\mathrm{NaBiF}_{4}$ : Gd@PDA@PEG nanoparticles had promising potential for T1\&MRTI dual-modality MRI guided PTT.

\section{T1\&MRTI performance in vivo}

Subsequently, T1\&MRTI dual-modality MRI was conducted for PTT in vivo. After NaBiF4: Gd@PDA@PEG nanoparticles were intratumorally injected into mice

(Figure 3A-B), the region of tumor was immediately lit up. From the pseudo-color images, the distribution of nanoPTAs could be observed more clearly (Figure 3C-D). The results indicated that the nanomaterials had shortened $\mathrm{T} 1$, which could be utilized to localize tumor and track PTAs. Then, the T1 value in the region of interest (ROI) was measured in pre and post. Obviously, after injection of the nanomaterials, the T1 signal value increased significantly from 11.58 to 20.27 (Figure S14), which further demonstrated the nanomaterials had outstanding T1-weighted MRI performance.

After locating the nanomaterials, the irradiation experiments were carried out. Simultaneously, MRTI was utilized to record the real-time temperature change. As shown in Figure 3E-F, the temperature mapping and real-time variation in tumor area were recorded during $342 \mathrm{~s}$ irradiation. The maximum temperature change could reach to $12 \mathrm{~K}$. By comparison, when only irradiation was implemented, the tumor's 
temperature changed barely (Figure S15). Many literatures indicated the desired effective temperature of thermal therapy located around $43-45{ }^{\circ} \mathrm{C}^{44-47}$, therefore, it was increasingly necessary to monitor the real-time variation during this temperature range. As shown in Figure 4A, the real-time and accurate temperature change ranging between 6 and 10 degrees was recorded. In addition, the time resolution was as high as 19 seconds per frame, which ensured the parameters (illumination intensity and time, etc.) of PTT could be adjusted in time, thus leading to tremendous improvement of accuracy for PTT. It was feasible and satisfactory to dynamically observe the tumor temperature variation in different ROIs utilizing MRTI. As described in Figure 4B-C, the temperature of different ROIs in tumor increased along with irradiation time, which contributed to monitoring the treatment of tumor in time.

In addition, it was of great significance to record the real-time temperature change in tumor and adjacent normal tissue, which was favorable to maximize thermal ablation of tumor and minimize damage to healthy tissue. The temperature change was investigated in three different ROIs (Figure 5), including ROI (1) in tumor, ROI (2) and ROI (3) in peritumor normal tissue. Compared to ROI (2) and ROI (3), the temperature of ROI (1) increased significantly, which meant this strategy was capable of improving PTT therapeutic effect and reducing thermal radiation damage to normal tissues. The temperature resolution between adjacent time intervals could be as low as $0.1 \mathrm{~K}$, ensuring the accuracy of temperature change. As a result, on a single MRI machine, dual-modality MRI (T1/MRTI) could be adopted to localize PTAs and 
monitor the real-time temperature change during PTT to improve the accuracy of PTT.

\section{Monitoring temperature without soft tissue depth limitation by MRTI}

The capability of temperature monitoring between infrared thermal camera and magnetic resonance imaging was next compared. The association between temperature and depth in vivo by MRTI was firstly studied. From Figure S16, temperature change in vivo at different depths $(0-12 \mathrm{~mm})$ was measured accurately. Comparatively, infrared thermal camera was used to imitatively record the temperature change in various depths. As shown in Figure S17, infrared thermal camera was utilized to measure the temperature change of $\mathrm{NaBiF}_{4}$ : Gd@PDA@PEG solution covered with different thickness $(0,0.25,0.5,1.0,2.0,3.0 \mathrm{~cm})$ of pork during irradiation. Without pork tissue, camera had the ability to real-time record the temperature fluctuation. However, infrared thermal camera couldn't accurately reflect the temperature change even with the thinnest pork thickness $(2.5 \mathrm{~mm})$. In a word, MRTI could be qualified for monitoring the real-time temperature change in vivo with little soft tissue depth limitation compared to infrared thermal camera.

\section{Toxicity and biocompatibility of $\mathrm{NaBiF}_{4}$ : Gd@PDA@PEG nanoparticles in vivo}

In consideration of clinical application of $\mathrm{NaBiF}_{4}$ : Gd@PDA@PEG, its toxicity and biocompatibility in vivo were investigated. $100 \mu \mathrm{L}$ of $\mathrm{NaBiF}_{4}$ : Gd@PDA@PEG (10000 ppm, Bi) solution or PBS (control group) was intravenously into healthy mice for short-term (3 days) and long-term (30 days) potential toxicity consideration. After 
a month's careful examination, there was no significant difference in body weight between the experimental group and the control group (Figure S18). Moreover, hematoxylin and eosin (H\&E) results also demonstrated the safety of heart, liver, spleen, lung and kidney (Figure S19). The tests of serum biochemistry and hematology further validated the outstanding biocompatibility and low toxicity of NaBiF 4 : Gd@PDA@PEG (Figure S20). Then, the mice digital images were acquired to indicate the photothermal capability of $\mathrm{NaBiF}_{4}$ : Gd@PDA@PEG to kill tumor after PTT (Figure S21). H\&E staining of tumor demonstrated large areas of necrosis appeared after photothermal therapy (Figure S22). In addition, the pharmacokinetic property of $\mathrm{NaBiF}_{4}$ : Gd@PDA@PEG was reflected by its half-life of blood $\left(\mathrm{t}_{0.5}\right)$ of mice (Figure S23), which was calculated as $76 \mathrm{~min}$. And its biodistribution revealed the nanomaterials were mainly accumulated in the liver and spleen (Figure S24).

In conclusion, an innovative strategy was proposed to integrate T1-MRI with MRTI for efficiently evaluating the spatial location of nanoPTAs, and accurately monitoring the real-time temperature change in vivo during PTT on a single MRI machine. The strategy could reveal the spatial temperature change with high accuracy, and further ensure the effective ablation of tumor with minimal damage to normal tissue. We demonstrated that $\mathrm{NaBiF}_{4}: \mathrm{Gd} @$ PDA@PEG as nanoPTAs had favorable biocompatibility. T1-weighted properties (the longitudinal relaxation rate $\left(\mathrm{r}_{1}\right)$ of 1.2 $\mathrm{mM}^{-1} \mathrm{~s}^{-1}$ ) on an $11.7 \mathrm{~T}$ ultra-high field MRI machine made it possible to locate the distribution of nanoPTAs accurately, further giving detailed structural information of tumor. In addition, excellent photothermal conversion performance enabled $\mathrm{NaBiF}_{4}$ : 
Gd@PDA@PEG nanomaterials to be highly effective in eradicating tumors. Simultaneously, NaBiF 4 : Gd@PDA@PEG had exceedingly weak susceptibility $\left(1.04 * 10^{-6} \mathrm{emu} \mathrm{g}^{-1} \mathrm{Oe}^{-1}\right)$ and little interference to local phase. Therefore, MRTI had the capability to dynamically record real-time temperature change in tumor and adjacent normal tissue. The time resolution could reach to 19 seconds per frame and the detection accuracy of temperature change was about $0.1 \mathrm{~K}$. On a single machine, integrating anatomic with functional MRI for guiding PTT was of great significance to eliminate thermal damage to normal tissue and improve PTT accuracy, which further promoted the clinical development, such as interventional PTT surgery. 
SCHEME.

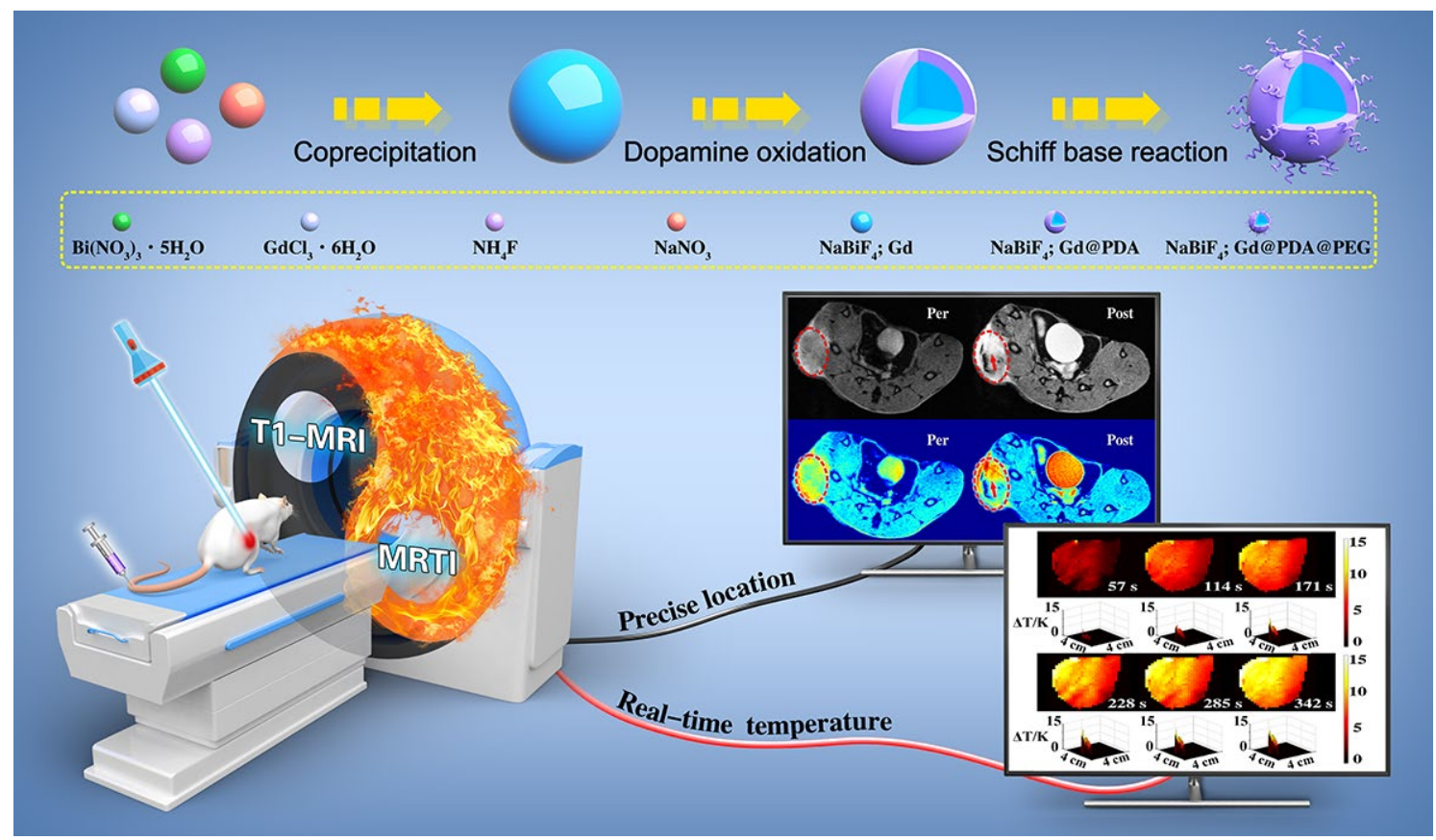

Scheme 1. Scheme of integrated MRI for guiding photothermal therapy. NanoPTAs with outstanding photothermal conversion performance and T1-weighted capability were synthesized. On a single $11.7 \mathrm{~T}$ magnetic machine, anatomic MRI (T1-MRI) was utilized to track nanoPTAs and exhibit more detailed structure information in tumor, meanwhile, functional MRI (MRTI) was conducted to accurately record the real-time temperature change in vivo. 


\section{FIGURES.}
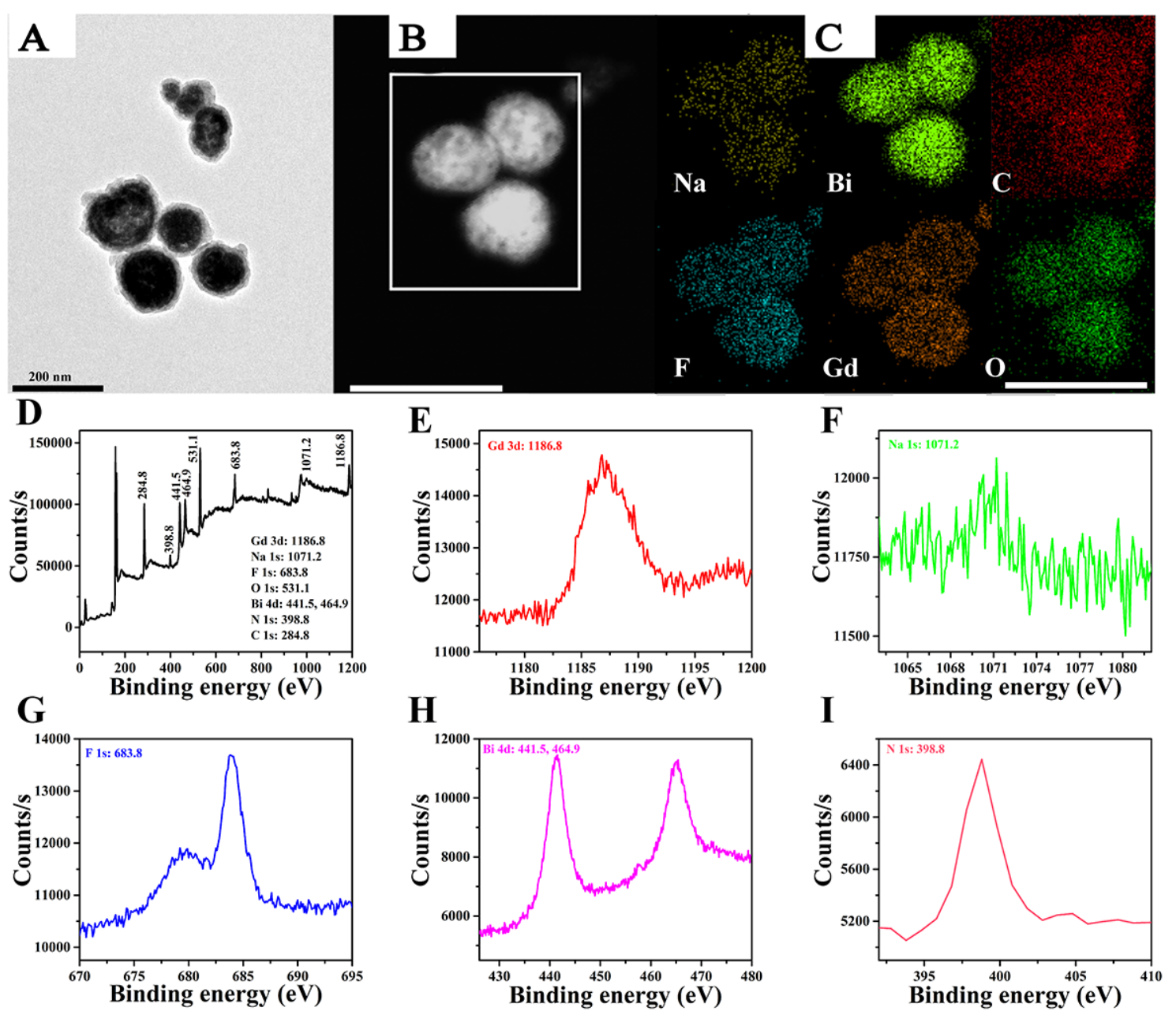

Figure 1. TEM images of $\mathrm{NaBiF}_{4}: \mathrm{Gd} @ \mathrm{PDA} @ \mathrm{PEG}(\mathrm{A})$; High-angle annular dark field image of $\mathrm{NaBiF}_{4}$ : Gd@PDA@PEG (B); The element mapping (Na, Bi, F, Gd, C, O) images of $\mathrm{NaBiF}_{4}$ : Gd@PDA@PEG (C). Scale bars were 200 nm. The XPS spectra of $\mathrm{NaBiF}_{4}: \mathrm{Gd} @$ PDA@PEG (D), Gd 3d: 1186.8 (E), Na 1s: 1071.2 (F), F 1s: 683.8 (G), Bi 4d: 441.5, $464.9(\mathrm{H}), \mathrm{N}$ 1s: 398.8 (I). 
A
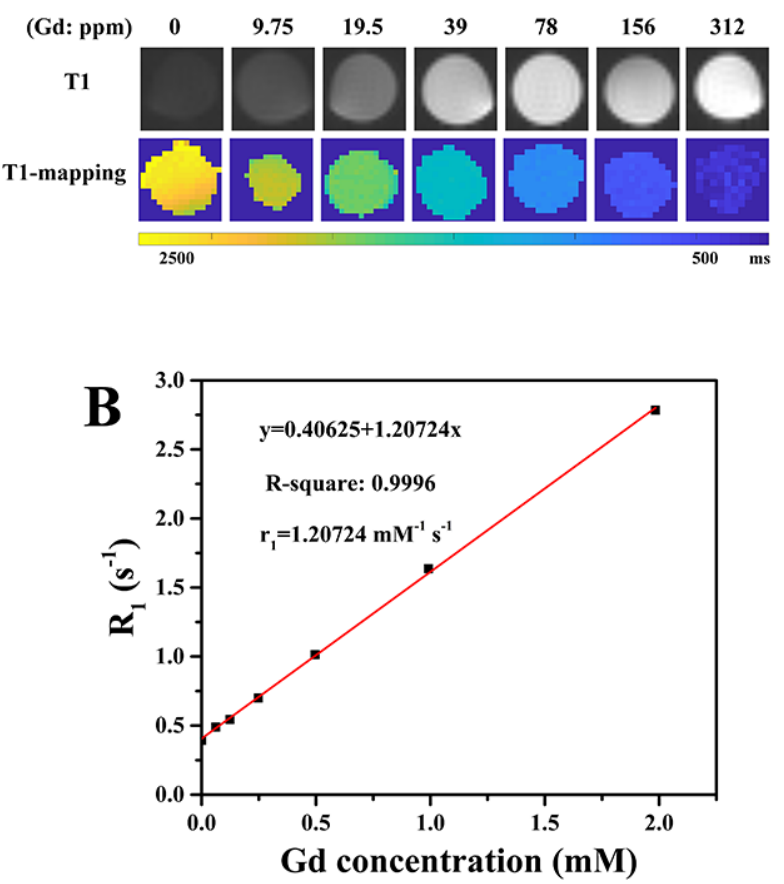
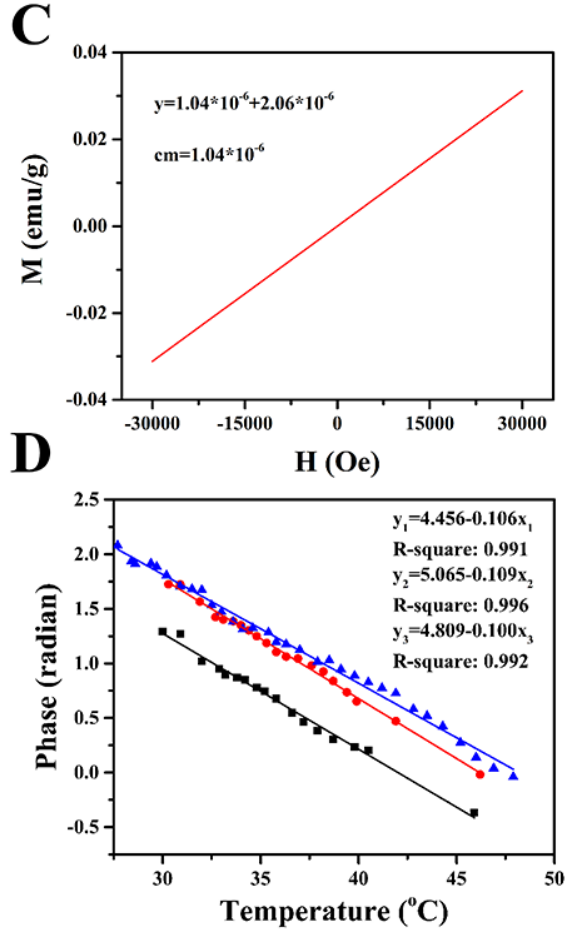

Figure 2. $\mathrm{T} 1$ weighted gray images and $\mathrm{T} 1$ mapping images of $\mathrm{NaBiF}_{4}$ :

Gd@PDA@PEG nanoparticles in different concentration of Gd (A); the responding longitudinal relaxtion $\left(\mathrm{R}_{1}\right)$ against the concentration of $\mathrm{Gd}(\mathrm{B})$; Hysteresis loops of NaBiF4:Gd@PDA@PEG nanoparticles at $300 \mathrm{~K}$ of temperature and $3 \mathrm{~T}$ of magnetic intensity (C); Phase changed along with temperature in agar with $\mathrm{NaBiF}_{4}$ : Gd@PDA@PEG of different Gd concentration (0, 30,60 ppm). Blue line (Gd, 0 ppm), black line (Gd, 30 ppm), red line (Gd, 60 ppm) (D). 


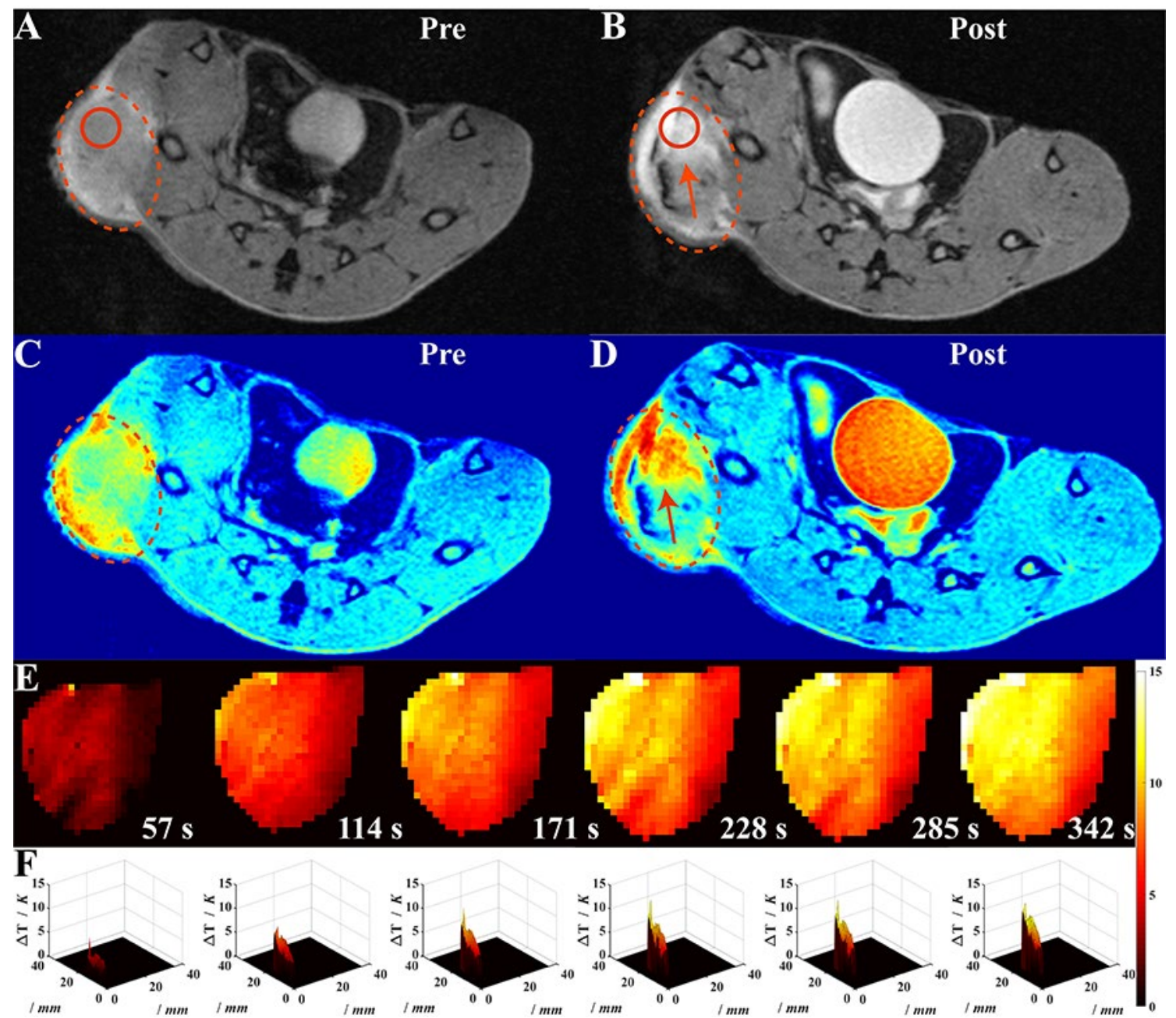

Figure 3. T1 weighted MRI images before (A, gray; C, pseudo color) and after (B, gray; D, pseudo color) intratumor injection of $\mathrm{NaBiF}_{4}$ : Gd@PDA@PEG (Gd, 61.5 ppm, Bi, $800 \mathrm{ppm}, 100 \mu \mathrm{L})$. Real-time temperature monitoring of tumors during $342 \mathrm{~s}$ of photothermal therapy after intratumor injection of $\mathrm{NaBiF}_{4}$ : Gd@PDA@PEG. Time interval was $57 \mathrm{~s}$, the 2-D (E) and 3-D (F) temperature mapping images were acquired. The area of dashed line was tumor and that of arrow was T1 signal enhancement. Then the T1 value of ROI (solid line) in pre and post was compared. 
A
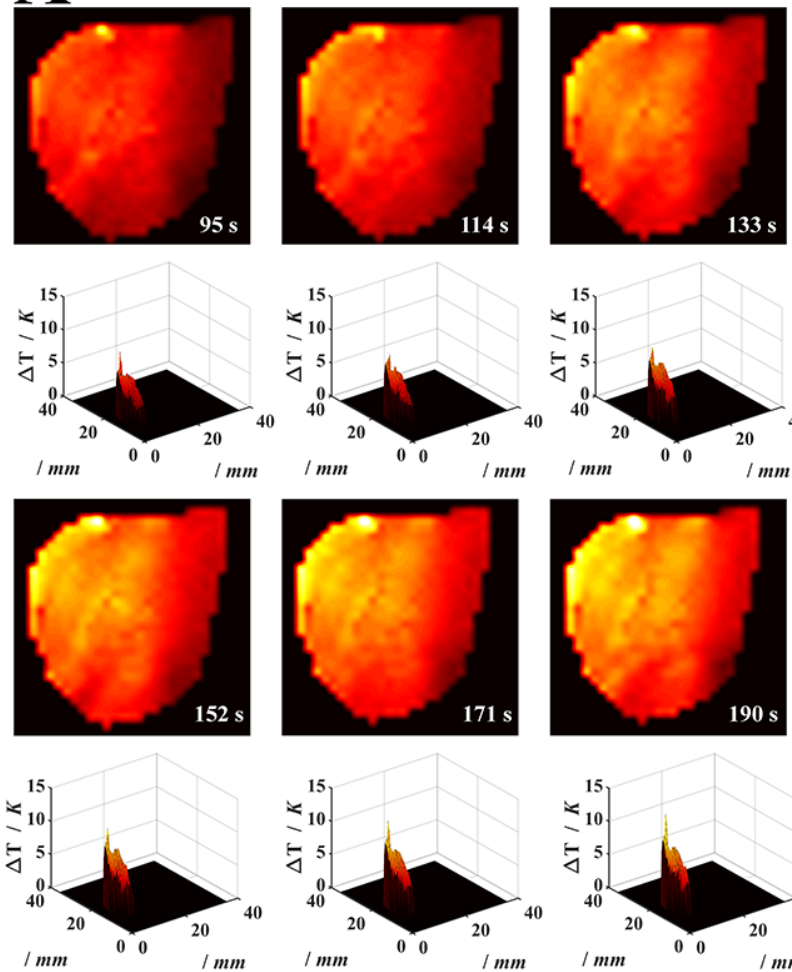
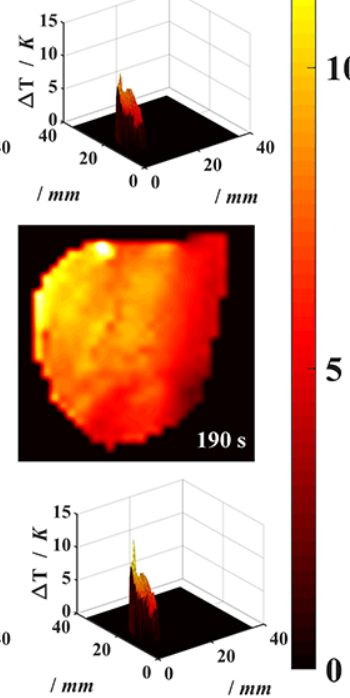

B
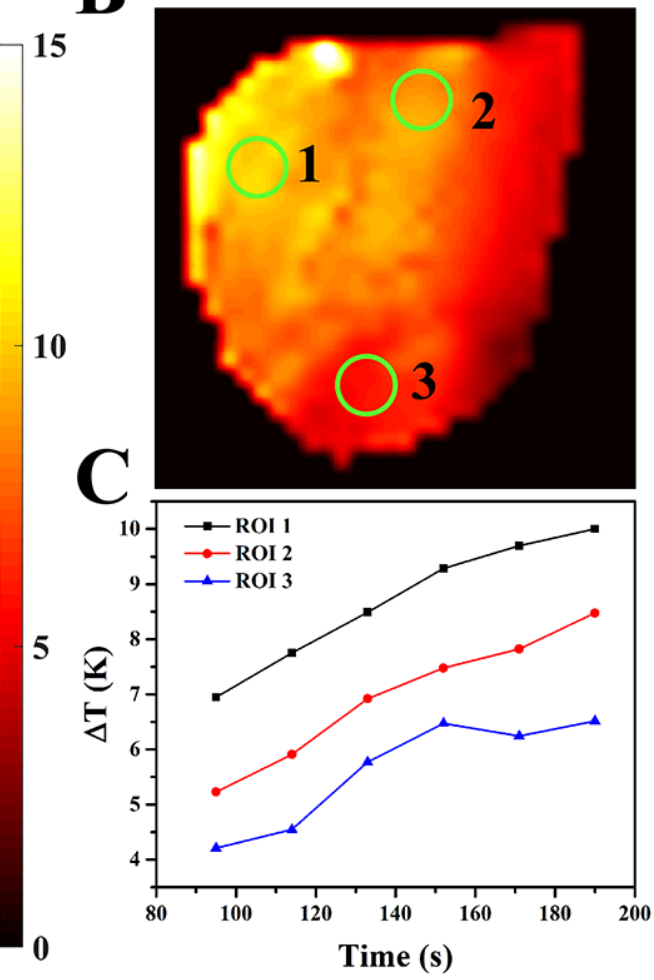

Figure 4. Real-time temperature monitoring of tumors of PTT after intratumor injection at time interval of $20 \mathrm{~s}$, and the 2-D and 3-D temperature mapping images were acquired. Three different ROI (B) and the corresponding temperature change (C). 

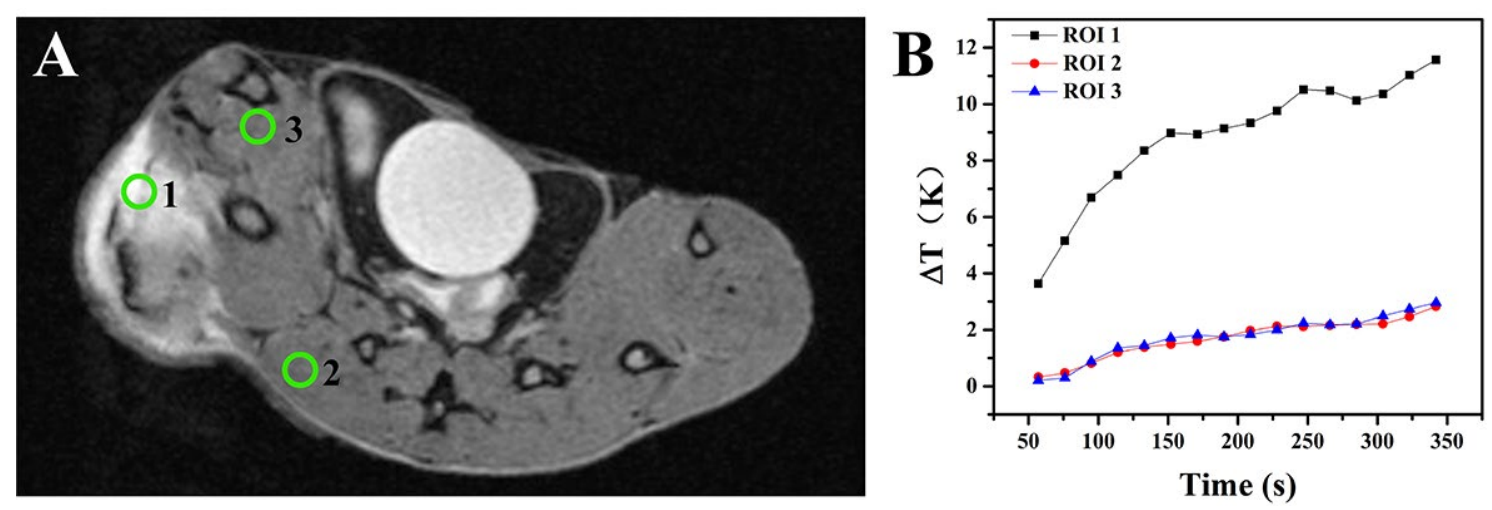

Figure 5. T1 images after intratumor injection of $\mathrm{NaBiF}_{4}$ : Gd@PDA@PEG (A). And the temperature change in three ROI was acquired and compared during PTT. 1: tumor; 2 and 3: normal tissue near tumor. The corresponding temperature variation in 3 different ROI (B). The time interval was $20 \mathrm{~s}$. 
ASSOCIATED CONTENT

Supporting Information.

\section{AUTHOR INFORMATION}

\section{Corresponding Author}

*E-mail: hewang@fudan.edu.cn,wbbu@chem.ecnu.edu.cn

\section{Author Contributions}

†These authors contributed equally.

\section{ACKNOWLEDGMENT}

The authors would greatly acknowledge the financial support from the National Funds

for Distinguished Young Scientists (Grant No. 51725202), the Key Project of Shanghai Science and Technology Commission (Grant No. 19JC1412000), the National Natural Science Foundation of China (Grant No. 51872094, 81971583), the National Science Foundation for the Young Scientists of China (Grant No. 51702211, 21805090), Shanghai Municipal Science and Technology Major Project (Grant No. 2018SHZDZX01) and ZJLab, and Shanghai Natural Science Foundation (Grant No. 17ZR1401600).

\section{REFERENCES}

1. Huang, X.; ElSayed, I. H.; Qian, W.; El Sayed, M. A., et al. Cancer cell imaging and photothermal therapy in the near-infrared region by using gold nanorods. 2006, 
128 (6), 2115-2120.

2. Yang, K.; Zhang, S.; Zhang, G.; Sun, X.; Lee, S. T.; Liu, Z., et al. Graphene in mice: ultrahigh in vivo tumor uptake and efficient photothermal therapy. 2010, 10 (9), 3318-3323.

3. Robinson, J. T.; Tabakman, S. M.; Liang, Y.; Wang, H.; Sanchez Casalongue, H.; Vinh, D., et al. Ultrasmall reduced graphene oxide with high near-infrared absorbance for photothermal therapy. 2011, 133 (17), 6825-6831.

4. Cheng, L.; Yang, K.; Li, Y.; Chen, J.; Wang, C.; Shao, M.; Lee, S. T., et al. Facile preparation of multifunctional upconversion nanoprobes for multimodal imaging and dual-targeted photothermal therapy. 2011, 50 (32), 7385-7390.

5. Qin, Z., et al. Thermophysical and biological responses of gold nanoparticle laser heating. 2012, 41 (3), 1191-1217.

6. Liu, Y.; Bhattarai, P.; Dai, Z.; Chen, X., et al. Photothermal therapy and photoacoustic imaging via nanotheranostics in fighting cancer. 2019, 48 (7), 2053-2108.

7. Guo, R.; Zhang, L.; Qian, H.; Li, R.; Jiang, X.; Liu, B., et al. Multifunctional nanocarriers for cell imaging, drug delivery, and near-IR photothermal therapy. 2010, 26 (8), 5428-5434.

8. Moon, H. K.; Lee, S. H.; Choi, H. C., et al. In vivo near-infrared mediated tumor destruction by photothermal effect of carbon nanotubes. 2009, 3 (11), 3707-3713.

9. Chen, Q.; Hu, Q.; Dukhovlinova, E.; Chen, G.; Ahn, S., et al. Photothermal therapy promotes tumor infiltration and antitumor activity of CAR T cells. 2019, 
1900192.

10. Chen, Y.; Fan, Z.; Zhang, Z.; Niu, W.; Li, C.; Yang, N.; Chen, B., et al. Two-dimensional metal nanomaterials: synthesis, properties, and applications. 2018, 118 (13), 6409-6455.

11. Cheng, L.; Wang, C.; Feng, L., et al. Functional nanomaterials for phototherapies of cancer. 2014, 114 (21), 10869-10939.

12. Hong, G.; Diao, S.; Antaris, A. L., et al. Carbon nanomaterials for biological imaging and nanomedicinal therapy. 2015, 115 (19), 10816-10906.

13. Jaque, D.; Maestro, L. M.; Del Rosal, B.; Haro Gonzalez, P.; Benayas, A.; Plaza, J., et al. Nanoparticles for photothermal therapies. 2014, 6 (16), 9494-9530.

14. Shanmugam, V.; Selvakumar, S., et al. Near-infrared light-responsive nanomaterials in cancer therapeutics. 2014, 43 (17), 6254-6287.

15. Liu, X., et al. Heavily-doped colloidal semiconductor and metal oxide nanocrystals: an emerging new class of plasmonic nanomaterials. 2014, 43 (11), 3908-3920.

16. Huang, P.; Lin, J.; Li, W.; Rong, P., et al. Biodegradable gold nanovesicles with an ultrastrong plasmonic coupling effect for photoacoustic imaging and photothermal therapy. 2013, 52 (52), 13958-13964.

17. Liang, C.; Diao, S.; Wang, C.; Gong, H.; Liu, T.; Hong, G., et al. Tumor metastasis inhibition by imaging-guided photothermal therapy with single-walled carbon nanotubes. 2014, 26 (32), 5646-5652.

18. Qiu, M.; Wang, D.; Liang, W.; Liu, L.; Zhang, Y., et al. Novel concept of the 
smart NIR-light-controlled drug release of black phosphorus nanostructure for cancer therapy. 2018, $115(3), 501-506$.

19. Zhu, X.; Li, J.; Qiu, X., et al. Upconversion nanocomposite for programming combination cancer therapy by precise control of microscopic temperature. 2018, 9 (1), 2176.

20. Gabano, E.; Colangelo, D., et al. The influence of temperature on antiproliferative effects, cellular uptake and DNA platination of the clinically employed Pt (II)-drugs. 2008, $102(4), 629-635$.

21. Storm, F. K.; Harrison, W. H.; Elliott, R. S., et al. Normal tissue and solid tumor effects of hyperthermia in animal models and clinical trials. 1979, 39 (6 Part 2), 2245-2251.

22. Yatvin, M. B., et al. Related Studies in Physics, C.; Medicine, The influence of membrane lipid composition and procaine on hyperthermic death of cells. 1977, 32 (6), 513-521.

23. Lu, Y.; Xu, Y. J.; Zhang, G.; Ling, D.; Wang, M.; Zhou, Y.; Wu, Y. D.; Wu, T.; Hackett, M. J.; Kim, B. H., Iron oxide nanoclusters for T1 magnetic resonance imaging of non-human primates. 2017, 1 (8).

24. Morris, S. A., et al. Magnetic resonance imaging. 2018, 104-108.

25. Fütterer, J. J.; Briganti, A.; De Visschere, P., et al. Can clinically significant prostate cancer be detected with multiparametric magnetic resonance imaging? A systematic review of the literature. 2015, 68 (6), 1045-1053.

26. Debette, S.; Schilling, S., et al. Clinical significance of magnetic resonance 
imaging markers of vascular brain injury: a systematic review and meta-analysis. 2019, $76(1), 81-94$.

27. Estelrich, J.; Sánchez Martín, M. J., et al. Nanoparticles in magnetic resonance imaging: from simple to dual contrast agents. 2015, 10, 1727.

28. Shen, A.; Meng, X.; Gao, X.; Xu, X.; Shao, C., et al. An Adaptable Nanoplatform for Integrating Anatomic and Functional Magnetic Resonance Imaging under a 3.0 T Magnetic Field. 2019, 29 (2), 1803832.

29. Shao, C.; Shen, A.; Zhang, M.; Meng, X.; Song, C.; Liu, Y., et al. Oxygen Vacancies Enhanced $\mathrm{CeO}_{2}$ : $\mathrm{Gd}$ Nanoparticles for Sensing a Tumor Vascular Microenvironment by Magnetic Resonance Imaging. 2018, 12 (12), 12629-12637.

30. Ni, D.; Shen, Z.; Zhang, J.; Zhang, C.; Wu, R.; Liu, J.; Yi, M., et al. Integrating anatomic and functional dual-mode magnetic resonance imaging: design and applicability of a bifunctional contrast agent. 2016, 10 (3), 3783-3790.

31. Zhu, M.; Sun, Z., et al. surgery, Image-guided thermal ablation with MR-based thermometry. 2017, 7 (3), 356.

32. Winter, L.; Oberacker, E.; Paul, K.; Ji, Y., et al. Magnetic resonance thermometry: methodology, pitfalls and practical solutions. 2016, 32 (1), 63-75.

33. Nick, T.; Mahamadou, D., et al. In vivo evaluation of multi-echo hybrid PRF/T1 approach for temperature monitoring during breast MR-guided focused ultrasound surgery treatments. 2015, 72 (3), 793-799.

34. Sprinkhuizen, S. M.; Konings, M. K., et al. Temperature-induced tissue susceptibility changes lead to significant temperature errors in PRFS-based MR 
thermometry during thermal interventions. 2010, 64 (5), 1360-1372.

35. Rieke, V. J., et al. MR Thermometry. 2010, 27 (2), 376-390.

36. Poorter, J. D., et al. Noninvasive MRI thermometry with the proton resonance frequency method: Study of susceptibility effects. 2010, 34 (3), 359-367.

37. Bizzi, A., .; Brooks, R. A., et al. Role of iron and ferritin in MR imaging of the brain: a study in primates at different field strengths. 1990, 177 (1), 59-65.

38. Lei, P.; An, R.; Yao, S.; Wang, Q.; Dong, L., et al. Ultrafast Synthesis of Novel Hexagonal Phase $\mathrm{NaBiF}_{4}$ Upconversion Nanoparticles at Room Temperature. 2017, $29(22), 1700505$.

39. Lu, C.; Li, Y.; Wang, L., et al. Rational design of two-dimensional covalent tilings using a C6-symmetric building block via on-surface Schiff base reaction. 2018, 55 (9).

40. Zhang, H.; Li, Y.; Pan, Z.; Chen, Y., et al. Multifunctional nanosystem based on graphene oxide for synergesitic multistage tumor-targeting and combined chemo-photothermal therapy.

41. Lin, L. S.; Cong, Z. X.; Cao, J. B., et al. Multifunctional $\mathrm{Fe}_{3} \mathrm{O}_{4} @$ Polydopamine Core-Shell Nanocomposites for Intracellular mRNA Detection and Imaging-Guided Photothermal Therapy. 2014, 8 (4), 3876.

42. Liu, Y.; Ai, K.; Lu, L., Polydopamine and Its Derivative Materials: Synthesis and Promising Applications in Energy, Environmental, and Biomedical Fields.

43. Lynge, M. E.; Westen, R. V. D., et al. Polydopamine-a nature-inspired polymer coating for biomedical science. 2011, 3 . 
44. Hauck, T. S.; Jennings, T. L.; Yatsenko, T., et al. Enhancing the toxicity of cancer chemotherapeutics with gold nanorod hyperthermia. 2008, 20 (20), 3832-3838.

45. Hankiewicz, J.; Celinski, Z., et al. Ferromagnetic particles as magnetic resonance imaging temperature sensors. 2016, 7, 12415.

46. Coffey, D. S.; Getzenberg, R. H., et al. Hyperthermic biology and cancer therapies: a hypothesis for the "Lance Armstrong effect". 2006, 296 (4), 445-448.

47. Wust, P.; Hildebrandt, B.; Sreenivasa, G., et al. Hyperthermia in combined treatment of cancer. 2002, $3(8), 487-497$. 